\title{
mRNA-1273 COVID-19 vaccine effectiveness against the B.1.1.7 and B.1.351 variants and severe COVID-19 disease in Qatar
}

\author{
Hiam Chemaitelly ${ }^{1,2}$, Hadi M. Yassine ${ }^{3,4}$, Fatiha M. Benslimane ${ }^{3,4}$, Hebah A. Al Khatib ${ }^{3,4}$, \\ Patrick Tang ${ }^{5}$, Mohammad R. Hasan $\mathbb{1}^{5}$, Joel A. Malek ${ }^{6,7}$, Peter Coyle ${ }^{3,8,9}$, Houssein H. Ayoub ${ }^{10}$, \\ Zaina Al Kanaani ${ }^{8}$, Einas Al Kuwari ${ }^{8}$, Andrew Jeremijenko ${ }^{8}{ }^{8}$, Anvar Hassan Kaleeckal ${ }^{8}$, \\ Ali Nizar Latif ${ }^{8}$, Riyazuddin Mohammad Shaik ${ }^{8}$, Hanan F. Abdul Rahim ${ }^{11}$, Gheyath K. Nasrallah ${ }^{3,4}$, \\ Mohamed Ghaith Al Kuwari' ${ }^{12}$, Hamad Eid Al Romaihi ${ }^{13}$, Mohamed H. Al-Thani ${ }^{13}$, Abdullatif Al Khal ${ }^{8}$, \\ Adeel A. Butt ${ }^{8,14}$, Roberto Bertollini ${ }^{13}$ and Laith J. Abu-Raddad (1) 1,2,14凶
}

The SARS-CoV-2 pandemic continues to be a global health concern. The mRNA-1273 (Moderna) vaccine was reported to have an efficacy of $94.1 \%$ at preventing symptomatic COVID-19 due to infection with 'wild-type' variants in a randomized clinical trial. Here, we assess the real-world effectiveness of this vaccine against SARS-CoV-2 variants of concern, specifically B.1.1.7 (Alpha) and B.1.351 (Beta), in Qatar, a population that comprises mainly working-age adults, using a matched test-negative, case-control study design. We show that vaccine effectiveness was negligible for $\mathbf{2}$ weeks after the first dose, but increased rapidly in the third and fourth weeks immediately before administration of a second dose. Effectiveness against B.1.1.7 infection was $88.1 \%$ (95\% confidence interval $(\mathrm{CI}): \mathbf{8 3 . 7 - 9 1 . 5 \% )} \geq \mathbf{1 4}$ days after the first dose but before the second dose, and was $100 \%$ (95\% $\mathrm{Cl}: 91.8-$ $100.0 \%) \geq 14$ days after the second dose. Analogous effectiveness against B.1.351 infection was $61.3 \%$ after the first dose (95\% Cl: 56.5-65.5\%) and 96.4\% after the second dose (95\% Cl: 91.9-98.7\%). Effectiveness against any severe, critical or fatal COVID-19 disease due to any SARS-CoV-2 infection (predominantly B.1.1.7 and B.1.351) was 81.6\% (95\% Cl: 71.0-88.8\%) and 95.7\% (95\% Cl: 73.4-99.9\%) after the first and second dose, respectively. The mRNA-1273 vaccine is highly effective against B.1.1.7 and B.1.351 infections, whether symptomatic or asymptomatic, and against any COVID-19 hospitalization and death, even after a single dose.

n a randomized clinical trial, the mRNA-1273 (Moderna) vaccine had a reported efficacy of $94.1 \%$ in preventing symptomatic coronavirus disease 2019 (COVID-19) due to infection with wild-type variants $^{1}$. The first immunization using this vaccine in Qatar was recorded on 28 December 2020 , but mass vaccination did not start until late February 2021 with the accelerated arrival of vaccine shipments. As the vaccination campaign was scaled up, the country experienced two back-to-back severe acute respiratory syndrome coronavirus 2 (SARS-CoV-2) waves that were triggered by the introduction and expansion of the B.1.1.7 (Alpha $\left.{ }^{2}\right)$ and B.1.351 $\left(\right.$ Beta $\left.^{2}\right)$ variants $^{3}$. This created a unique epidemiological opportunity to assess the real-world effectiveness of this vaccine against infection with these variants of concern, as well as against severe forms of COVID-19 disease.

Results

Study population. Between 28 December 2020 and 10 May 2021, 256,037 individuals in Qatar received at least one dose of the mRNA1273 vaccine and 181,304 completed the two-dose regimen. Among those with a vaccination record, the median dates at first and second dose were 5 and 29 April 2021, respectively. The median time elapsing between the first and second doses was 28 days (interquartile range (IQR): $28-29$ days), with $94.6 \%$ of individuals receiving their second dose $\leq 30$ days after the first dose.

Flowcharts describing the population selection process for investigation and estimation of vaccine effectiveness are presented in Extended Data Figs. 1-3, and the demographic characteristics of the sample for each outcome of vaccine effectiveness are presented in Table 1. Median age in the study sample for estimation of vaccine effectiveness against infection with B.1.1.7 was 31 years (IQR: 19-38), against infection with B.1.351 was 32 years (IQR: 25-39) and against severe, critical or fatal disease was 43 years (IQR: 37-51). Of note is that Qatar has a young and diverse demographic, where only $9 \%$ of its resident population are $>50$ years of age and $89 \%$ are expatriates from $>150$ countries $^{4,5}$.

Weekly rounds of viral genome sequencing from 8 March to 10 May 2021 identified B.1.351 $(n=369 ; 64.4 \%)$, B.1.1.7 $(n=58$;

'Infectious Disease Epidemiology Group, Weill Cornell Medicine-Qatar, Cornell University, Qatar Foundation-Education City, Doha, Qatar. ${ }^{2}$ World Health Organization Collaborating Centre for Disease Epidemiology Analytics on HIV/AIDS, Sexually Transmitted Infections, and Viral Hepatitis, Weill Cornell Medicine-Qatar, Cornell University, Qatar Foundation-Education City, Doha, Qatar. ${ }^{3}$ Biomedical Research Center, Member of QU Health, Qatar University, Doha, Qatar. ${ }^{4}$ Department of Biomedical Science, College of Health Sciences, Member of QU Health, Qatar University, Doha, Qatar. ${ }^{5}$ Department of Pathology, Sidra Medicine, Doha, Qatar. ${ }^{6}$ Genomics Laboratory, Weill Cornell Medicine-Qatar, Cornell University, Doha, Qatar. ${ }^{7}$ Department of Genetic Medicine, Weill Cornell Medicine-Qatar, Cornell University, Doha, Qatar. ${ }^{8}$ Hamad Medical Corporation, Doha, Qatar. ${ }^{9}$ Wellcome-Wolfson Institute for Experimental Medicine, Queens University, Belfast, UK. ${ }^{10}$ Department of Mathematics, Statistics, and Physics, Qatar University, Doha, Qatar. "'College of Health Sciences, QU Health, Qatar University, Doha, Qatar. ${ }^{12}$ Primary Health Care Corporation, Doha, Qatar. ${ }^{13}$ Ministry of Public Health, Doha, Qatar. ${ }^{14}$ Department of Population Health Sciences, Weill Cornell Medicine, Cornell University, New York, NY, USA. 凶e-mail: lja2002@qatar-med.cornell.edu 
Table 1 | Demographic characteristics of (1) B.1.1.7 cases (PCR-positive) and controls (PCR-negative), (2) B.1.351 cases (PCRpositive) and controls (PCR-negative) and (3) severe, critical or fatal COVID-19 disease cases (any PCR-positive case) and controls (PCR-negative)

\begin{tabular}{|c|c|c|c|c|c|c|}
\hline \multirow{2}{*}{$\begin{array}{l}\text { Sample type } \\
\text { Characteristics }^{\mathrm{a}}\end{array}$} & \multicolumn{2}{|c|}{$\begin{array}{l}\text { (1) Any infection with the B.1.1.7 variant } \\
\text { between } 1 \text { February and } 10 \text { May } 2021\end{array}$} & \multicolumn{2}{|c|}{$\begin{array}{l}\text { (2) Any infection with the B.1.351 variant } \\
\text { between } 8 \text { March and } 10 \text { May } 2021\end{array}$} & \multicolumn{2}{|c|}{$\begin{array}{l}\text { (3) Any severe, critical or fatal disease } \\
\text { with any SARS-CoV-2 infection } \\
\text { between } 1 \text { February and } 10 \text { May } 2021\end{array}$} \\
\hline & $\begin{array}{l}\text { Cases }^{\mathrm{b}} \\
\text { (PCR-positive) }\end{array}$ & $\begin{array}{l}\text { Controls }^{\mathrm{b}} \\
\text { (PCR-negative) }\end{array}$ & $\begin{array}{l}\text { Cases }^{\mathrm{b}} \\
\text { (PCR-positive) }\end{array}$ & $\begin{array}{l}\text { Controls } \\
\text { (PCR-negative) }\end{array}$ & $\begin{array}{l}\text { Cases }^{\mathrm{b}} \\
\text { (PCR-positive) }\end{array}$ & $\begin{array}{l}\text { Controls }{ }^{\mathrm{b}} \\
\text { (PCR-negative) }\end{array}$ \\
\hline $\begin{array}{l}\text { Median age, years } \\
\text { (IQR) }\end{array}$ & $31(19-38)$ & $31(19-38)$ & $32(25-39)$ & $32(25-39)$ & $43(37-51)$ & $43(37-51)$ \\
\hline $30-39$ years & $6,885(32.3)$ & $6,885(32.3)$ & $16,652(37.2)$ & $16,652(37.2)$ & $1,011(29.8)$ & $1,011(29.8)$ \\
\hline $40-49$ years & $3,330(15.6)$ & $3,330(15.6)$ & $7,857(17.6)$ & $7,857(17.6)$ & $1,224(36.1)$ & $1,224(36.1)$ \\
\hline $50-59$ years & $1,042(4.9)$ & $1,042(4.9)$ & $2,025(4.5)$ & $2,025(4.5)$ & $687(20.2)$ & $687(20.2)$ \\
\hline $60-69$ years & $230(1.1)$ & $230(1.1)$ & $338(0.8)$ & $338(0.8)$ & $203(6.0)$ & $203(6.0)$ \\
\hline \multicolumn{7}{|l|}{ Nationalityc } \\
\hline Bangladeshi & $903(4.2)$ & $903(4.2)$ & $3,687(8.2)$ & $3,687(8.2)$ & $364(10.7)$ & $364(10.7)$ \\
\hline Egyptian & $1,240(5.8)$ & $1,240(5.8)$ & $1,953(4.4)$ & $1,953(4.4)$ & $169(5.0)$ & $169(5.0)$ \\
\hline Filipino & $2,911(13.7)$ & $2,911(13.7)$ & $4,583(10.2)$ & 4,583 (10.2) & 507 (14.9) & 507 (14.9) \\
\hline Indian & $4,676(22.0)$ & $4,676(22.0)$ & 15,235 (34.1) & 15,235 (34.1) & $827(24.4)$ & $827(24.4)$ \\
\hline Nepalese & $1,159(5.4)$ & $1,159(5.4)$ & $5,251(11.7)$ & $5,251(11.7)$ & 407 (12.0) & 407 (12.0) \\
\hline Pakistani & $1,453(6.8)$ & $1,453(6.8)$ & $1,771(4.0)$ & $1,771(4.0)$ & $188(5.5)$ & $188(5.5)$ \\
\hline Qatari & $3,171(14.9)$ & $3,171(14.9)$ & $4,019(9.0)$ & $4,019(9.0)$ & $284(8.4)$ & $284(8.4)$ \\
\hline Sri Lankan & $617(2.9)$ & 617 (2.9) & $1,700(3.8)$ & $1,700(3.8)$ & $136(4.0)$ & $136(4.0)$ \\
\hline
\end{tabular}

${ }^{\mathrm{a} T}$ These demographic characteristics are for samples used in the $\geq 14$-days-after-second-dose analyses, but are essentially identical to those in the other analyses. ${ }^{\mathrm{b}} \mathrm{Cases}$ and controls were matched one-to-one by sex, age, nationality and reason for PCR testing. 'Nationalities were chosen to represent the most populous groups in Qatar. ${ }^{\mathrm{D}}$ These comprise 80 other nationalities in Qatar among sample 1 , 78 other nationalities among sample 2 and 41 other nationalities among sample 3.

$10.1 \%)$, B.1.617 $(n=18 ; 3.1 \%)$ and wild-type/undetermined variants $(n=128 ; 22.3 \%)$ in 573 randomly collected polymerase chain reaction (PCR)-positive specimens. Weekly rounds of multiplex quantitative PCR with reverse transcription (RT-qPCR)-based variant screening ${ }^{6}$ from 23 March to 10 May 2021 identified B.1.351-like $(n=2,605 ; 66.4 \%)$, B.1.1.7-like $(n=970 ; 24.7 \%)$ and 'other' variants $(n=349 ; 8.9 \%)$ in 3,924 randomly collected PCR-positive specimens. Sanger sequencing of the receptor-binding domain of SARS-CoV-2 spike protein on 109 other specimens confirmed that 103 were B.1.617-like, three were B.1-like and three were undetermined.

As of 10 May 2021, 1,558 and 243 breakthrough infections were recorded among those who had received one or two doses of the mRNA-1273 vaccine, respectively. Seven COVID-19 deaths were also recorded among mRNA-1273 vaccine recipients, all after the first dose and none after the second.

Vaccine effectiveness against infection with B.1.1.7. Estimated effectiveness against infection with B.1.1.7, defined as a PCR-positive swab with the B.1.1.7 variant, regardless of the reason for PCR testing or presence of symptoms (Methods), was negligible for 2 weeks after the first dose (Table 2 and Fig. 1), but increased rapidly in the third week reaching $81.6 \%$ (95\% CI: 73.1-87.8\%). Effectiveness was $94.4 \%$ (95\% CI: $89.1-97.5 \%$ ) in the fourth week immediately before the second dose, and reached 99.2\% (95\% CI: 95.3-100.0\%) in the second week after the second dose. Effectiveness was $88.1 \%$ (95\% CI: 83.7-91.5\%) 14 or more days after the first dose but before receiving the second dose, and was 100\% (95\% CI: 91.8-100.0\%) 14 or more days after the second dose (Table 3 ).

Vaccine effectiveness against infection with B.1.351. Effectiveness against infection with B.1.351, defined as a PCR-positive swab with the B.1.351 variant regardless of the reason for PCR testing or presence of symptoms (Methods), was negligible for 2 weeks after the first dose (Table 2 and Fig. 1) but increased rapidly in the third week to reach $47.9 \%$ (95\% CI: 39.5-55.2\%). Effectiveness was $73.7 \%$ (95\% CI: 67.6-78.8\%) in the fourth week immediately before the second dose, and reached 96.4\% (95\% CI: 94.3-97.9\%) in the second week after the second dose. Effectiveness was $61.3 \%$ (95\% CI: 56.5-65.5\%) 14 or more days after the first dose but before receiving the second dose, and was $96.4 \%$ (95\% CI: 91.9-98.7\%) 14 or more days after the second dose (Table 3 ).

Vaccine effectiveness against COVID-19 severity or fatality. Effectiveness against any severe, critical or fatal disease due to any SARS-CoV-2 infection (predominantly B.1.1.7 and B.1.351 (ref. ${ }^{7}$ ); 


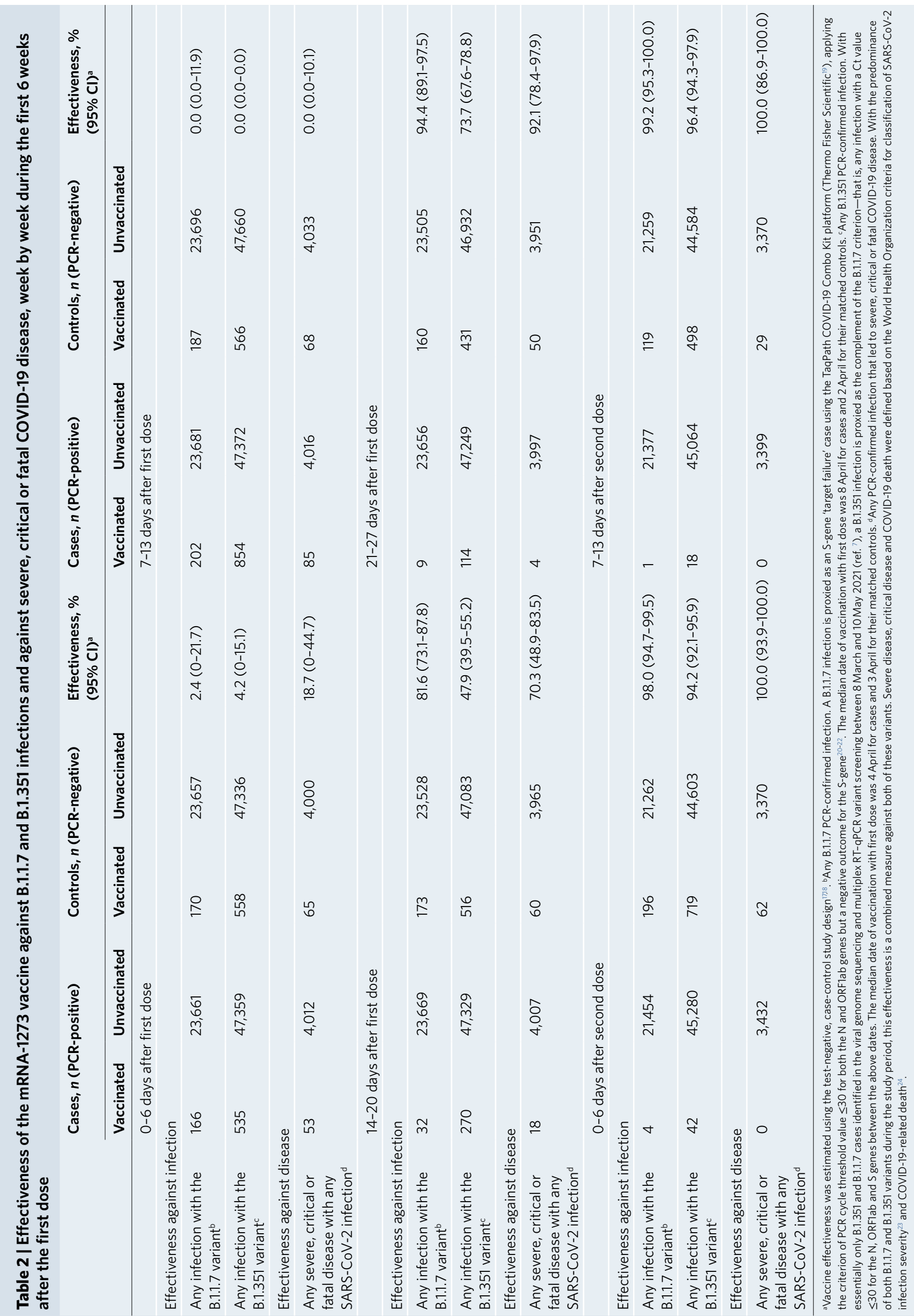



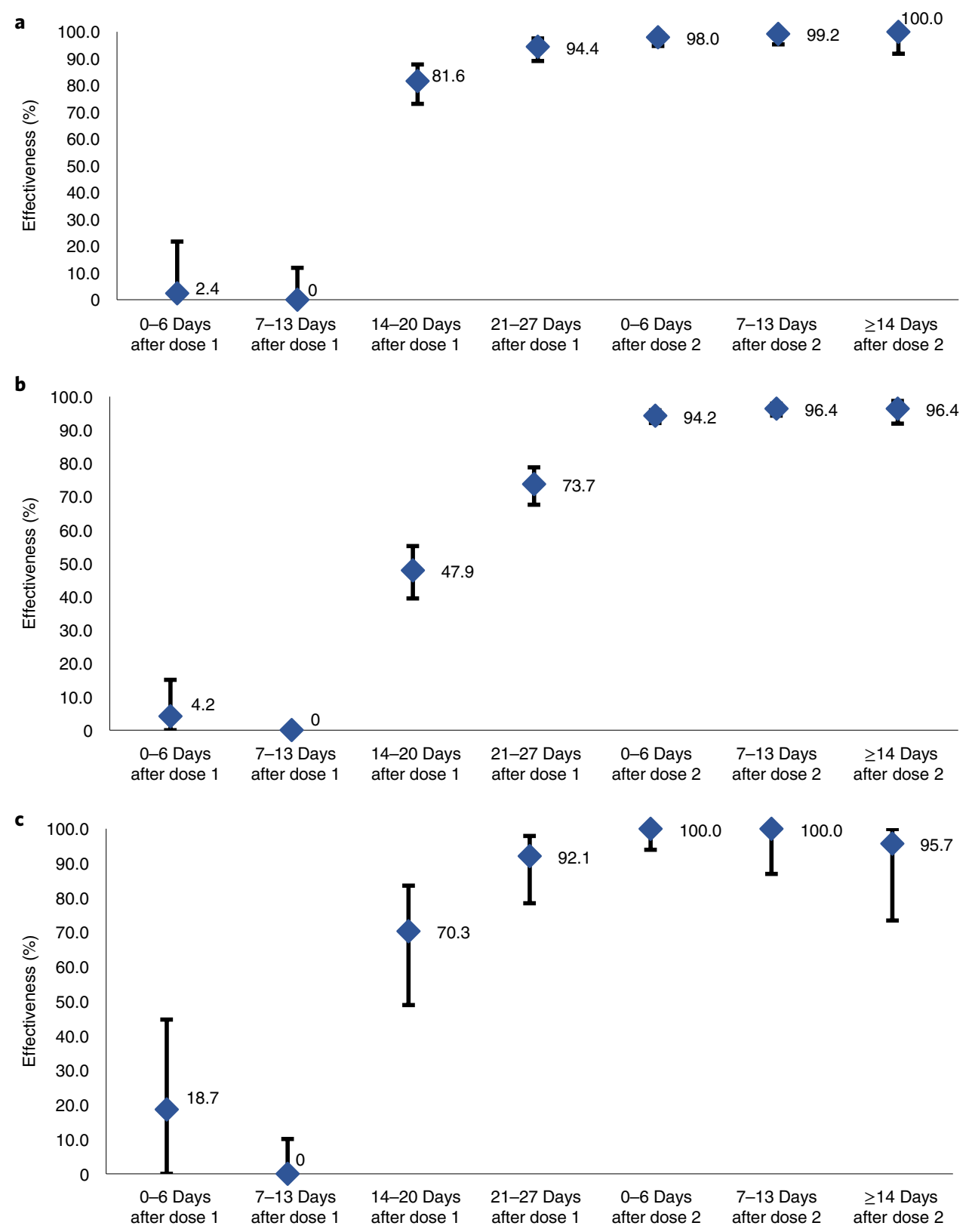

Fig. 1 | Effectiveness of the mRNA-1273 vaccine. a-c, Effectiveness of the mRNA-1273 vaccine against B.1.1.7 infections (a), B.1.351 infections (b) and severe, critical or fatal COVID-19 disease (c) after the first and second doses. Analyses were performed on independent samples of $n=25,034$ PCR-positive cases and $n=25,034$ PCR-negative controls examined between 1February and 10 May 2021 for B.1.1.7, $n=52,442$ PCR-positive cases and $n=52,442$ PCR-negative controls examined between 8 March and 10 May 2021 for B.1.351, $n=4,497$ PCR-positive cases that progressed to severe, critical or fatal disease and $n=4,497$ PCR-negative controls examined between 1 February and 10 May 2021. Data are presented as effectiveness point estimates, with error bars indicating the corresponding $95 \% \mathrm{Cls}$.

Methods) was negligible for 2 weeks after the first dose (Table 2 and Fig. 1) but increased rapidly in the third week to reach $70.3 \%(95 \%$ CI: $48.9-83.5 \%)$. Effectiveness was $92.1 \%$ (95\% CI: 78.4-97.9\%) in the fourth week immediately before the second dose, and reached $100 \%$ (95\% CI: 86.9-100.0\%) in the second week after the second dose. Effectiveness was $81.6 \%$ (95\% CI: $71.0-88.8 \%$ ) 14 or more days after the first dose but before receiving the second dose, and was $95.7 \%$ (95\% CI: 73.4-99.9\%) 14 or more days after the second dose (Table 3).

Additional analyses. Sensitivity analyses matching by PCR testing date in addition to age, sex, nationality and reason for PCR testing; adjusting for calendar week in logistic regression; or additionally adjusting for matching factors-that is, sex, age, nationality and reason for PCR testing, all confirmed the main analysis results (Table 4).

An additional analysis estimated vaccine effectiveness against symptomatic infection (predominantly B.1.1.7 and B.1.351 (ref. ${ }^{7}$ )) at $98.6 \%$ (95\% CI: $92.0-100.0 \%) 14$ or more days after the second dose (Supplementary Table 1). Symptomatic infection was defined as a PCR-positive test conducted because of clinical suspicion due to the presence of symptoms compatible with a respiratory tract infection.

An additional analysis estimated vaccine effectiveness against asymptomatic infection (predominantly B.1.1.7 and B.1.351 ${ }^{7}$ ) at 92.5\% (95\% CI: $84.8-96.9 \%) 14$ or more days after the second dose 


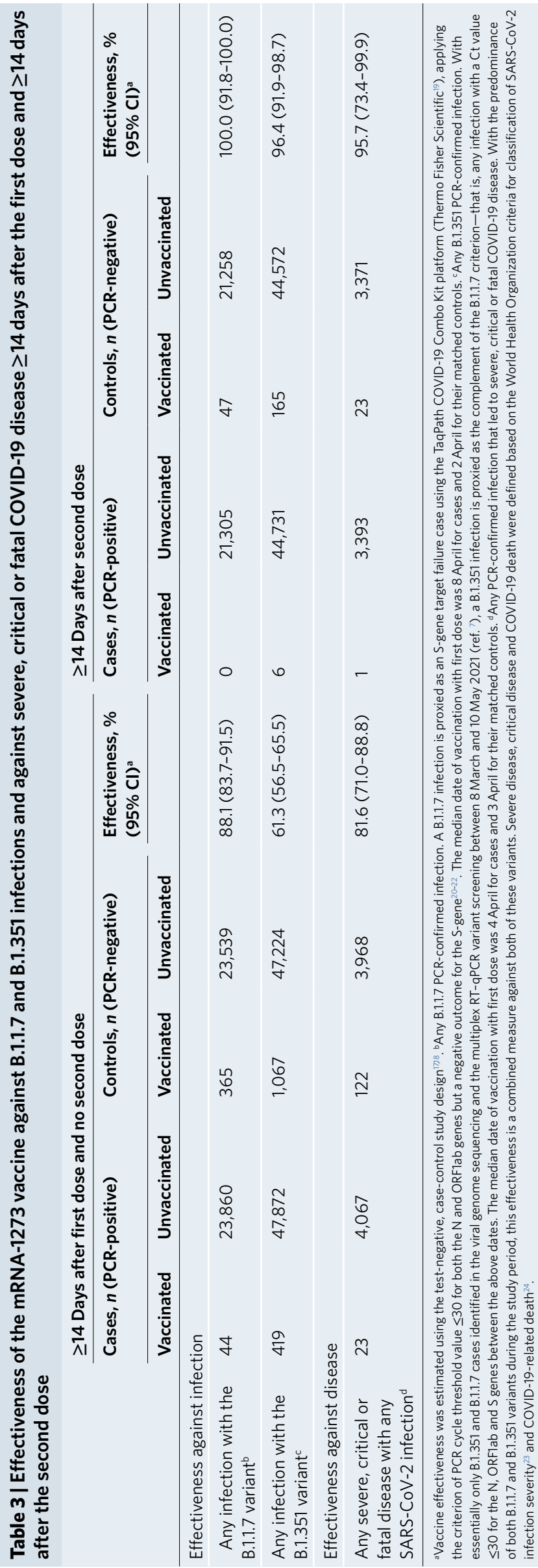

(Supplementary Table 1). Asymptomatic infection was defined as a PCR-positive test conducted with no reported presence of symptoms compatible with a respiratory tract infection-that is, PCR testing was done as part of a survey, for pretravel requirements or at a port of entry following arrival in the country ${ }^{4,8}$.

An additional analysis estimated vaccine effectiveness 14 or more days after the second dose using a cohort study design that compares infection incidence in those vaccinated with that in the national cohort of persons who were antibody negative (Methods, Supplementary Table 2 and Extended Data Fig. 4). Incidence rates among those vaccinated were estimated at 0 (95\% CI: $0-3.60)$ for B.1.1.7, 5.83 (95\% CI: 2.62-12.99) for B.1.351 and 1.94 (95\% CI: 0.49-7.78) for variants of unknown status per 10,000 person-weeks in a total follow-up time of 10,282.86 person-weeks. The incidence rate among those antibody negative was estimated at 20.49 (95\% CI: 19.40-21.63) for B.1.1.7, 47.78 (95\% CI: 46.11-49.52) for B.1.351 and 30.12 (95\% CI: 28.79-31.50) for variants of unknown status per 10,000 person-weeks in a total follow-up time of 631,171.10 person-weeks. Vaccine effectiveness was thus estimated at $100.0 \%$ (95\% CI: $82.5-100.0 \%$ ) against B.1.1.7, 87.8\% (95\% CI: 73.4-95.5\%) against B.1.351 and 93.5\% (95\% CI: 76.6-99.2\%) against variants of unknown status, supporting the results from the main analysis.

\section{Discussion}

Our analysis demonstrates that the effectiveness of the mRNA1273 vaccine is high, regardless of variant, against both symptomatic and asymptomatic infection, and against COVID-19 hospitalization and death. Development of vaccine protection against B.1.1.7 infection and COVID-19 hospitalization and death rapidly accelerated in the third and fourth weeks after the first dose, immediately before the second dose, nearly reaching the values attained after the second dose. Protection against B.1.351 infection exhibited a similar pattern, but mounted at a slower rate and did not achieve its highest value of $~ 95 \%$ until after the second dose. While long-term protection of only one dose could not be assessed beyond 4 weeks after the first dose, these findings might suggest that most of the protection from this vaccine is attained using only one dose, apart possibly from its protection against B.1.351. Although for optimal protection the protocol for this vaccine requires a second dose 28 days after the first, given the sizable protection achieved after only one dose, in situations involving limited vaccine supplies, these findings may support a strategy of delaying the second dose to vaccinate the largest number of people in the shortest time, as supported also by evidence for the BNT162b2 (Pfizer-BioNTech) and ChAdOx1 nCoV-19 (AZD1222; Oxford-AstraZeneca) vaccines ${ }^{9-11}$. Having said that, the optimal timing of the second dose is best determined formally through randomized clinical trials and could also depend on both the incidence level and circulating variants, which are factors that can vary from one setting to another.

The mRNA-1273 vaccine demonstrated similar levels and patterns of protection after the first and second doses to the BNT162b2 vaccine, as estimated recently in Qatar ${ }^{3,11}$. However, in our study, the mRNA-1273 vaccine appears to offer greater protection against both B.1.351 and B.1.1.7. It is premature to conclude that this outcome is due to a superior vaccine-induced immune response, as other confounding variables may also have contributed to observed differences. The second dose of mRNA-1273 is administered 28 days after the first, 1 week later than for BNT162b2, which might have affected the build-up of immunity and estimated effectiveness. We also note that since the mass immunization campaigns in Qatar started with BNT162b2 in December 2020, most older persons and those with comorbid conditions were vaccinated with the BNT162b2 vaccine before the mRNA-1273 vaccine became widely available in March-May 2021. Those vaccinated with the mRNA-1273 vaccine 


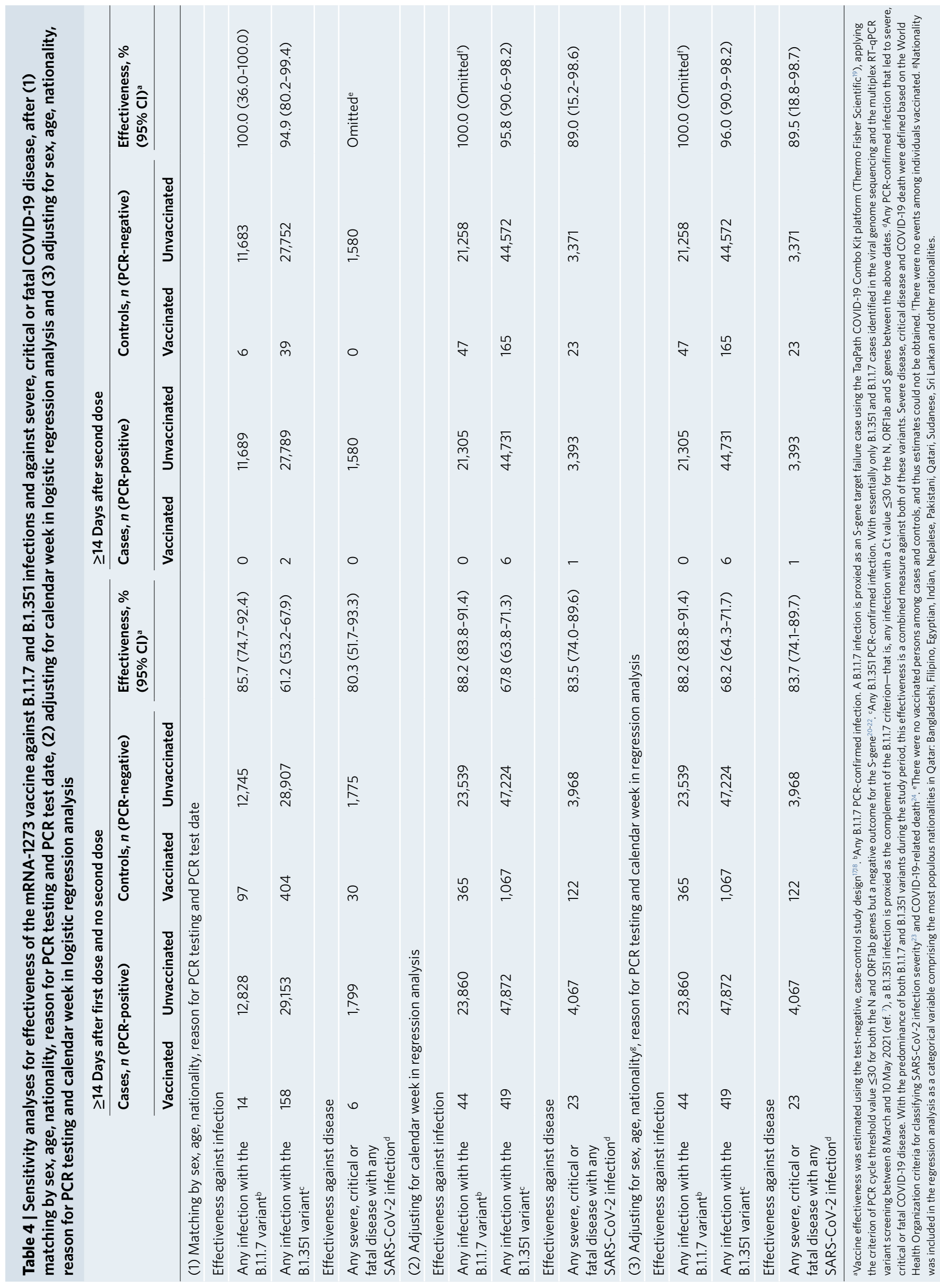


(Table 1 ) tend to be slightly younger than those vaccinated with the BNT162b2 vaccine ${ }^{3}$.

Effectiveness in our study was assessed based on documented infections, but other infections may have occurred and gone undocumented perhaps because of minimal and/or mild symptoms or by virtue of being subclinical infections. However, with the high rate of PCR testing in Qatar, 64.2\% of infections diagnosed during the study period were identified not because of clinical symptoms but for other reasons, including random testing campaigns (surveys), contact tracing, individual requests and routine healthcare- and travel-related testing. This indicates that the vaccine was robustly effective not only against symptomatic clinical disease, but also against asymptomatic and subclinical infection. That said, there was evidence for a slight gradient in vaccine effectiveness by appearance of symptoms - that is, greater protection against more symptomatic or severe infections, as observed for the BNT162b2 vaccine ${ }^{3,11}$.

This study has certain limitations. Estimated effectiveness against severe, critical or fatal COVID-19 disease had somewhat wider $95 \%$ confidence intervals as a consequence of (1) the relatively lower severity of COVID-19 in the young population of Qatar compared to populations where the median age is higher ${ }^{4,12},(2)$ the younger cohort of those vaccinated with mRNA-1273 and (3) the majority of vaccinated persons having had their second dose after the B.1.1.7 and B.1.351 waves peaked in early March and early April 2021 , respectively. The median date of receiving the second dose in our study population was 29 April 2021. For the same reasons, we could not precisely estimate effectiveness against severe, critical or fatal COVID-19 disease attributable individually to the B.1.1.7 and B.1.351 variants.

Severe, critical or fatal COVID-19 disease was assessed up to the end of the study (10 May 2021), and it is possible that cases confirmed as PCR positive immediately before the end of the study may have progressed to a severe outcome only after the end of the study and were thus not included in our sample. This, however, should not affect our estimates because the study, by design, contrasts confirmed COVID-19 disease cases with PCR-negative cases. For reasons that remain unclear, among persons 7-13 days after the first dose the risk of infection with B.1.351 was higher compared to those who remained unvaccinated, an outcome observed elsewhere for both COVID-19 vaccines ${ }^{13-15}$ and other vaccines ${ }^{16}$. This might reflect a higher underlying risk of infection, bias due to uncontrolled confounding such as differences in social behavior at or following vaccination, an immunological effect ${ }^{16}$ or an artifact of the estimation method, possibly because the first vaccine dose frequently coincided with the peak of the B.1.351 wave.

Imperfect assay sensitivity and specificity of PCR testing may also have affected infection ascertainment. However, all PCR testing was performed with extensively used, investigated and validated commercial platforms having essentially $100 \%$ sensitivity and specificity (Methods). The baseline analysis did not factor matching or control for calendar time, but sensitivity analyses factoring calendar time confirmed the main findings. Data on comorbid conditions were not available and hence could not be factored explicitly in our analysis. However, adjustment for age may have served as a proxy given that comorbidities are associated with old age. Furthermore, given the young population structure in Qatar ${ }^{4,5}$ we anticipate that only a small proportion of the study population may have had serious comorbid conditions. However, our findings might not be entirely generalizable to other settings where the elderly constitute a sizable proportion of the population. Effectiveness was assessed using an observational test-negative, case-control study design ${ }^{17,18}$ rather than a randomized clinical trial, but the cohort study design also yielded similar findings, supporting the validity of this approach in assessing vaccine effectiveness. In some instances there were zero events among cases and/or controls, thus precluding estimation of effectiveness and/or associated confidence intervals.
In conclusion, in the population of Qatar the mRNA-1273 vaccine is very effective against both B.1.1.7 and B.1.351 infection, whether symptomatic or asymptomatic, and against COVID-19 hospitalization and death. Most of the protection of this vaccine accrued in the third and fourth weeks after the first dose and before the second dose, although its effectiveness against B.1.351 increased substantially more after the second dose. These findings justify optimism that vaccine-induced immunity will prove robust against known variants of concern, thereby reducing the likelihood of a protracted pandemic for years to come.

\section{Online content}

Any methods, additional references, Nature Research reporting summaries, source data, extended data, supplementary information, acknowledgements, peer review information; details of author contributions and competing interests; and statements of data and code availability are available at https://doi.org/10.1038/ s41591-021-01446-y.

Received: 20 May 2021; Accepted: 22 June 2021;

Published online: 9 July 2021

\section{References}

1. Baden, L. R. et al. Efficacy and safety of the mRNA-1273 SARS-CoV-2 vaccine. N. Engl. J. Med. 384, 403-416 (2021).

2. Tracking SARS-CoV-2 variants (World Health Organization, accessed 5 June 2021); https://www.who.int/en/activities/tracking-SARS-CoV-2-variants/

3. Abu-Raddad, L. J., Chemaitelly, H., Butt, A. A. \& National Study Group for Covid-19 Vaccination. Effectiveness of the BNT162b2 Covid-19 vaccine against the B.1.1.7 and B.1.351 variants. N. Engl. J. Med. https://pubmed.ncbi. nlm.nih.gov/33951357/ (2021).

4. Abu-Raddad, L. J. et al. Characterizing the Qatar advanced-phase SARS-CoV-2 epidemic. Sci. Rep. 11, 6233 (2021).

5. Qatar Monthly Statistics (Planning and Statistics Authority, accessed 26 May 2020); https://www.psa.gov.qa/en/pages/default.aspx

6. Vogels, C., Fauver, J. \& Grubaugh, N. Multiplexed RT-qPCR to screen for SARS-COV-2 B.1.1.7, B.1.351, and P.1 variants of concern V.3. https://doi. org/10.17504/protocols.io.br9vm966 (2021).

7. National Project of Surveillance for Variants of Concern and Viral Genome Sequencing. Qatar viral genome sequencing data. Data on randomly collected samples between February 1-May 10, 2021 (Ministry of Public Health, 2021).

8. Bertollini, R. et al. Associations of vaccination and of prior infection with positive PCR test results for SARS-CoV-2 in airline passengers arriving in Qatar. JAMA https://pubmed.ncbi.nlm.nih.gov/34106201/ (2021).

9. Lopez Bernal, J. et al. Effectiveness of the Pfizer-BioNTech and OxfordAstraZeneca vaccines on Covid-19 related symptoms, hospital admissions, and mortality in older adults in England: test negative case-control study. Brit. Med. J. 373, n1088 (2021).

10. Voysey, M. et al. Single-dose administration and the influence of the timing of the booster dose on immunogenicity and efficacy of ChAdOxl nCoV-19 (AZD1222) vaccine: a pooled analysis of four randomised trials. Lancet 397, 881-891 (2021).

11. Abu-Raddad, L. J. et al. Pfizer-BioNTech mRNA BNT162b2 Covid-19 vaccine protection against variants of concern after one versus two doses. J. Travel Med. https://pubmed.ncbi.nlm.nih.gov/34050372/ (2021).

12. Seedat, S. et al. SARS-CoV-2 infection hospitalization, severity, criticality, and fatality rates. Preprint at https://www.medrxiv.org/content/10.1101/2020.11.29. 20240416v1.full (2020).

13. Hitchings, M. D. T. et al. Effectiveness of CoronaVac in the setting of high SARS-CoV-2 P.1 variant transmission in Brazil: a test-negative case-control study. Preprint at https://www.medrxiv.org/content/10.1101/2021.04.07.21255 081v1 (2021).

14. Hunter, P. R. \& Brainard, J. Estimating the effectiveness of the Pfizer COVID-19 BNT162b2 vaccine after a single dose. A reanalysis of a study of 'real-world' vaccination outcomes from Israel. Preprint at https://www. medrxiv.org/content/10.1101/2021.02.01.21250957v1 (2021).

15. Hall, V. J. et al. COVID-19 vaccine coverage in health-care workers in England and effectiveness of BNT162b2 mRNA vaccine against infection (SIREN): a prospective, multicentre, cohort study. Lancet 397, 1725-1735 (2021).

16. Daum, R. S., Siber, G. R., Ballanco, G. A. \& Sood, S. K. Serum anticapsular antibody response in the first week after immunization of adults and infants with the Haemophilus influenzae type b-Neisseria meningitidis outer membrane protein complex conjugate vaccine. J. Infect. Dis. 164, 1154-1159 (1991). 
17. Jackson, M. L. \& Nelson, J. C. The test-negative design for estimating influenza vaccine effectiveness. Vaccine 31, 2165-2168 (2013).

18. Verani, J. R. et al. Case-control vaccine effectiveness studies: preparation, design, and enrollment of cases and controls. Vaccine 35, 3295-3302 (2017).

19. Thermo Fisher Scientific. TaqPath ${ }^{\mathrm{ma}}$ COVID-19 CE-IVD RT-PCR Kit instructions for use (Thermo Fisher Scientific, accessed 2 December 2020); https://assets.thermofisher.com/TFS-Assets/LSG/manuals/MAN0019215_ TaqPathCOVID-19_CE-IVD_RT-PCR\%20Kit_IFU.pdf

20. Rapid increase of a SARS-CoV-2 variant with multiple spike protein mutations observed in the United Kingdom (ECDC, accessed 10 February 2021); https://www.ecdc.europa.eu/sites/default/files/documents/SARS-CoV2-variant-multiple-spike-protein-mutations-United-Kingdom.pdf

21. Galloway, S. E. et al. Emergence of SARS-CoV-2 B.1.1.7 Lineage - United States, December 29, 2020-January 12, 2021. MMWR Morb. Mortal. Wkly Rep. 70, 95-99 (2021).
22. Challen, R. et al. Risk of mortality in patients infected with SARS-CoV-2 variant of concern 202012/1: matched cohort study. Brit. Med. J. 372, n579 (2021)

23. COVID-19 clinical management: living guidance (WHO, accessed 15 May 2021); https://www.who.int/publications/i/item/ WHO-2019-nCoV-clinical-2021-1

24. International guidelines for certification and classification (coding) of COVID-19 as cause of death (WHO, accessed 15 May 2021); https://www. who.int/classifications/icd/Guidelines_Cause_of_Death_COVID-1920200420-EN.pdf?ua=1

Publisher's note Springer Nature remains neutral with regard to jurisdictional claims in published maps and institutional affiliations.

๑ The Author(s), under exclusive licence to Springer Nature America, Inc. 2021 


\section{Methods}

Data sources and study design. This study was conducted in the resident population of Qatar. COVID-19 laboratory testing, vaccination, clinical infection data and related demographic details were extracted from the integrated nationwide digital health information platform that hosts the national, federated SARS-CoV-2 databases. These databases are complete and have captured all SARS-CoV-2-related data since epidemic onset. Nearly all individuals were vaccinated (free of charge) in Qatar and not elsewhere. In rare situations where an individual had received the mRNA-1273 COVID-19 vaccine outside Qatar, the individual's vaccination details were still recorded in the health system at the port of entry (airport) following their return to Qatar given the national requirements and to benefit from privileges associated with vaccination, such as quarantine exemption.

Vaccine effectiveness was estimated using the test-negative, case-control study design, a standard design for assessment of vaccine effectiveness against influenza ${ }^{17,18}$. Key to this design is the control of bias arising from misclassification of infection and differences in healthcare-seeking behavior between vaccinated and unvaccinated individuals ${ }^{17,18}$. The STROBE checklist can be found in Supplementary Table 3. Cases and controls were matched one-to-one by sex, age, nationality and reason for SARS-CoV-2 PCR testing. Matching of cases and controls was performed to control for known differences in risk of exposure to the infection in Qatar ${ }^{4,25-27}$.

Effectiveness was estimated against documented infection (defined as a PCR-positive swab regardless of the reason for PCR testing or presence of symptoms) with the B.1.1.7 or B.1.351 variant, as well as against severe, critical or fatal disease due to any SARS-CoV-2 infection (predominantly B.1.1.7 and B.1.351 $\left(\right.$ ref. $\left.^{7}\right)$ ). Classification of COVID-19 case severity (acute care hospitalizations ${ }^{23}$, criticality (intensive care unit hospitalizations) ${ }^{23}$ and fatality ${ }^{24}$ followed the World Health Organization guidelines, and assessments were made by trained medical personnel using individual chart reviews.

Classification of infections by variant type was informed by weekly rounds of viral genome sequencing and multiplex RT- ${ }_{\text {PCR variant screening }}^{6}$ of randomly collected clinical samples ${ }^{7}$, as well as by results of deep sequencing of wastewater samples ${ }^{7}$. Based on existing evidence ${ }^{20-22}$, a B.1.1.7 case was defined as an S-gene target failure using TaqPath COVID-19 Combo Kits (Thermo Fisher Scientific ${ }^{19}$; $>85 \%$ of PCR testing in Qatar), applying the criterion of a PCR cycle threshold $(\mathrm{Ct})$ value $\leq 30$ for both the $\mathrm{N}$ and ORF1ab genes and a negative outcome for the S gene $^{22}$. Meanwhile, with essentially only B.1.351 and B.1.1.7 cases identified from 8 March 2021 until the end of the study (10 May 2021) in the viral genome sequencing and multiplex RT-qPCR variant screening ${ }^{7}$, a B.1.351 case was proxied as the complement of B.1.1.7 criteria-that is, any infection with a Ct value $\leq 30$ for the N, ORF1ab and $\mathrm{S}$ genes ${ }^{3}$.

With these considerations in regard to ascertaining infection by B.1.1.7 and B.1.351, the study extended from 1 February to 10 May 2021 for B.1.1.7, from 8 March to 10 May 2021 for B.1.351 and from 1 February to 10 May 2021 for any severe, critical or fatal COVID-19 disease. All records of PCR testing for those vaccinated and unvaccinated during the study duration were examined. All records of vaccination with one or two doses using a vaccine other than mRNA-1273 were excluded. Every case that met the inclusion criteria (a B.1.1.7 case, a B.1.351 case or a severe or critical or fatal COVID-19 disease case) and that could be matched to a control was included in the analysis. Both PCR test outcomes and vaccination status were ascertained at the time of the PCR test. Each person with a positive PCR test result and hospital admission was subject to an infection severity assessment every 3 days until discharge or death. Individuals who progressed to COVID-19 disease between the time of a positive PCR test result and the end of the study were classified based on their worst outcome, starting with death ${ }^{24}$, followed by critical disease ${ }^{23}$ and then severe disease ${ }^{23}$.

The study was approved by the Hamad Medical Corporation and Weill Cornell Medicine-Qatar Institutional Review Boards with waiver of informed consent.

Laboratory methods. Nasopharyngeal and/or oropharyngeal swabs (Huachenyang Technology) were collected for PCR testing and placed in Universal Transport Medium (UTM). Aliquots of UTM were extracted on a QIAsymphony platform (QIAGEN) and tested with RT-qPCR using TaqPath COVID-19 Combo Kits (100\% sensitivity and specificity ${ }^{19}$; Thermo Fisher Scientific) on an ABI 7500 FAST (ThermoFisher), extracted using a custom protocol $^{28}$ on a Hamilton Microlab STAR (Hamilton) and tested using AccuPower SARS-CoV-2 Real-Time RT-PCR Kits $\left(100 \%\right.$ sensitivity and specificity ${ }^{29}$; Bioneer) on an ABI 7500 FAST; or loaded directly into a Roche cobas 6800 system and assayed with a cobas SARS-CoV-2 Test (95\% sensitivity, $100 \%$ specificity ${ }^{30}$; Roche). The first assay targets the viral S, $\mathrm{N}$ and ORFlab regions, the second targets the viral RdRp and E-gene regions while the third targets the ORF1ab and E-gene regions.

All tests were conducted at either the HMC Central Laboratory or Sidra Medicine Laboratory, following standardized protocols.

Statistical analysis. Descriptive statistics (frequency distributions and measures of central tendency) were used to characterize study samples. The odds ratios, comparing odds of vaccination among cases to that among controls, and its associated $95 \%$ CI, were calculated using the exact method. Confidence intervals were not adjusted for multiplicity. Interactions were not investigated. Vaccine effectiveness at different time frames and its associated 95\% CI were then calculated by applying the following equation ${ }^{17,18}$ :

\section{Vaccine effectiveness$$
=1-\frac{\text { vaccinated among cases } \times \text { unvaccinated among controls }}{\text { vaccinated among controls } \times \text { unvaccinated among cases }}
$$

Additional analyses. To ensure that vaccine effectiveness estimates were not biased by epidemic phase and the gradual rollout of vaccination during the study, two sensitivity analyses were conducted, first matching by the exact PCR testing date and second by logistic regression to adjust for calendar week ${ }^{17,31}$. To further ensure control for confounding ${ }^{32,33}$, a third sensitivity analysis was conducted adjusting additionally for matching factors in logistic regression-that is sex, age, reason for PCR testing and nationality (grouping was based on the most populous nationality groups in Qatar; Tables 1 and 4 ).

Additional analyses were conducted to estimate vaccine effectiveness against symptomatic infection, defined as a PCR-positive test conducted because of clinical suspicion due to the presence of symptoms compatible with a respiratory tract infection, and against asymptomatic infection, defined as a PCR-positive test conducted with no reported presence of symptoms compatible with a respiratory tract infection-that is, PCR testing was done as part of a survey, for pretravel requirement or at the port of entry into the country (Supplementary Table 1).

Vaccine effectiveness was further assessed using a cohort study design $n^{3,34,35}$ by comparison of infection incidence in those who completed $\geq 14$ days after the second dose with incidence in the national cohort of individuals who were antibody negative. The incidence rate of infection was calculated for each of the variants using a Poisson log-likelihood regression model with the STATA 16.1 $\left(\right.$ ref. ${ }^{36}$ ) stptime command. The incidence rate ratio was calculated using the exact method. Vaccine effectiveness (with no adjustment for multiplicity) was estimated using the equation:

\section{Vaccine effectiveness

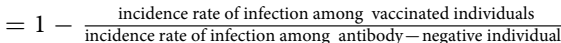

Further details on this type of analysis and the antibody-negative cohort can be found in our previous studies of reinfection ${ }^{34,35}$.

Analyses were conducted in STATA/SE $16.1\left(\right.$ ref. $^{36}$ ).

Reporting Summary. Further information on research design is available in the Nature Research Reporting Summary linked to this article.

\section{Data availability}

The dataset of this study is a property of the Qatar Ministry of Public Health and is provided to the researchers through a restricted-access agreement that prevents its sharing with a third party or publicly. Future access to this dataset can be considered through direct application for data access, to Her Excellency the Minister of Public Health (https://www.moph.gov.qa/english/Pages/default. aspx). Aggregate data are available within the manuscript and its Supplementary information.

\section{Code availability}

Standard epidemiological analyses were conducted using standard commands in STATA/SE 16.1 (ref. ${ }^{36}$ ). The commands/code are accessible using the following URL: https://github.com/IDEGWCMQ/Vaccine-effectiveness-code

\section{References}

25. Al-Thani, M. H. et al. SARS-CoV-2 infection is at herd immunity in the majority segment of the population of Qatar. Open Forum Infect. Dis. May 2, ofab221 (2021).

26. Jeremijenko, A. et al. Herd Immunity against severe acute respiratory syndrome coronavirus 2 infection in 10 communities, Qatar. Emerg. Infect. Dis. 27, 1343-1352 (2021)

27. Coyle, P. V. et al. SARS-CoV-2 seroprevalence in the urban population of Qatar: an analysis of antibody testing on a sample of 112,941 individuals. iScience 24, 102646 (2021).

28. Kalikiri, M. K. R. et al. High-throughput extraction of SARS-CoV-2 RNA from nasopharyngeal swabs using solid-phase reverse immobilization beads. Preprint at https://www.medrxiv.org/content/10.1101/2020.04.08.20055731v1 (2020).

29. Kubina, R. \& Dziedzic, A. Molecular and serological tests for COVID-19a comparative review of SARS-CoV-2 coronavirus laboratory and point-of-care diagnostics. Diagnostics (Basel) 10, 434 (2020).

30. Cobas ${ }^{\varpi}$ SARS-CoV-2: qualitative assay for use on the $\operatorname{cobas}^{\oplus} 6800 / 8800$ Systems (US FDA, accessed 2 December 2020); https://www.fda.gov/ media/136049/download

31. Jacoby, P. \& Kelly, H. Is it necessary to adjust for calendar time in a test negative design? Responding to: Jackson ML, Nelson JC. The test negative design for estimating influenza vaccine effectiveness. Vaccine 2013;31, 2165-8. Vaccine 32, 2942 (2014) 
32. Pearce, N. Analysis of matched case-control studies. Brit. Med. J. 352 , i969 (2016).

33. Rothman, K. J., Greenland, S. \& Lash, T. L. Modern Epidemiology (Wolters Kluwer Health/Lippincott Williams \& Wilkins, 2008).

34. Abu-Raddad, L. J. et al. Assessment of the risk of SARS-CoV-2 reinfection in an intense re-exposure setting. Clin. Infect. Dis. https://academic.oup.com/cid/ advance-article/doi/10.1093/cid/ciaa1846/6033728 (2020).

35. Abu-Raddad, L. J. et al. SARS-CoV-2 antibody-positivity protects against reinfection for at least seven months with 95\% efficacy. EClinicalMedicine 35, 100861 (2021).

36. Statistical Software: Release 16.1 (StataCorp, 2019); https://www.stata.com

\section{Acknowledgements}

We thank the many dedicated individuals at Hamad Medical Corporation, the Ministry of Public Health, the Primary Health Care Corporation and the Qatar Biobank for their diligent efforts and contributions to make this study possible. The authors are grateful for institutional salary support from the Biomedical Research Program, the Biostatistics, Epidemiology, and Biomathematics Research Core and the Genomics Core, all at Weill Cornell Medicine-Qatar, as well as for institutional salary support provided by the Ministry of Public Health and Hamad Medical Corporation. The authors also thank the Qatar Genome Program for institutional support in regard to the reagents needed for viral genome sequencing. The funders of the study had no role in study design, data collection, data analysis, data interpretation or writing of the article. Statements made herein are solely the responsibility of the authors.

\section{Author contributions}

H.C. codesigned the study, performed statistical analyses and cowrote the first draft of the article. L.J.A.-R. conceived and codesigned the study, led the statistical analyses and cowrote the first draft of the article. A.A.B. and R.B. codesigned the study. All authors contributed to data collection and acquisition, database development, discussion and interpretation of the results, and to the writing of the manuscript. All authors have read and approved the final manuscript.

\section{Competing interests}

A.A.B. received institutional grant funding from Gilead Sciences, unrelated to the work presented in this paper. Otherwise, the authors declare no competing interests.

\section{Additional information}

Extended data is available for this paper at https://doi.org/10.1038/s41591-021-01446-y. Supplementary information The online version contains supplementary material available at https://doi.org/10.1038/s41591-021-01446-y.

Correspondence and requests for materials should be addressed to L.J.A.-R.

Peer review information Nature Medicine thanks Mark Tenforde and the other, anonymous, reviewer(s) for their contribution to the peer review of this work. Jennifer Sargent was the primary editor on this article and managed its editorial process and peer review in collaboration with the rest of the editorial team.

Reprints and permissions information is available at www.nature.com/reprints. 
A)

161,788 individuals with a PCR-confirmed SARS-CoV-2 infection between February 1-May 10, 2021

24,547 individuals were excluded because they had a BNT162b2 vaccination record

137,241 individuals with a PCR-confirmed SARS-CoV-2 infection between February 1-May 10, 2021

25,990 individuals had a PCR-confirmed SARS-CoV-2 infection with the B.1.1.7 variant

25,034 individuals with a PCR-confirmed SARS-CoV-2 infection with the B.1.1.7 variant could be matched by age, sex, nationality, and reason for PCR testing to individuals with PCR-negative SARS-CoV-2 tests, for whom vaccination records were retrieved

444 individuals had at least one mRNA-1273 vaccine dose

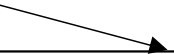

24,590 individuals had no vaccination record
891,081 individuals with PCR-negative SARS-CoV-2 tests between February 1-May 10, 2021

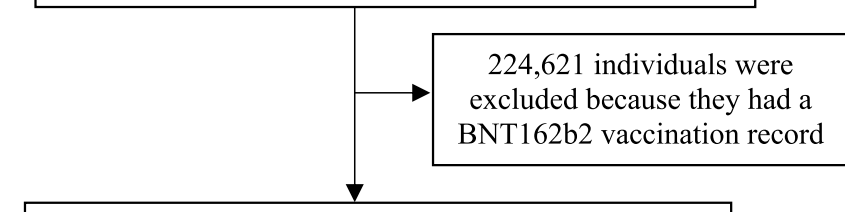

666,460 individuals with PCR-negative SARS-CoV-2 tests between February 1-May 10, 2021

Extended Data Fig. 1 | Flowcharts describing the population selection process to assess vaccine effectiveness against B.1.1.7. Flowcharts describing the population selection process for investigating mRNA-1273 vaccine effectiveness against infection with the B.1.1.7 variant. 
B)

161,788 individuals with a PCR-confirmed SARS-CoV-2 infection between February 1-May 10, 2021

24,547 individuals were excluded because they had a BNT162b2 vaccination record

137,241 individuals with a PCR-confirmed SARS-CoV-2 infection between February 1-May 10, 2021

29,101 individuals were excluded because they had the PCR test done before March 8, 2021

\section{8,140 individuals with a PCR-confirmed SARS-CoV-2 infection between March 8-May 10, 2021 eligible for inclusion in the analysis \\ 55,217 individuals had a PCR-confirmed SARS-CoV-2 infection with the B.1.351 variant}

52,442 individuals with a PCR-confirmed SARS-CoV-2 infection with the B.1.351 variant could be matched by age, sex, nationality, and reason for PCR testing to individuals with PCR-negative SARS-CoV-2 tests, for whom vaccination records were retrieved

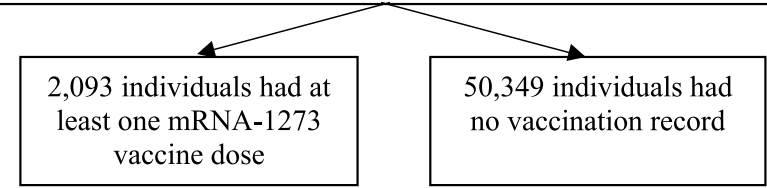

891,081 individuals with PCR-negative SARS-CoV-2 tests between February 1-May 10, 2021

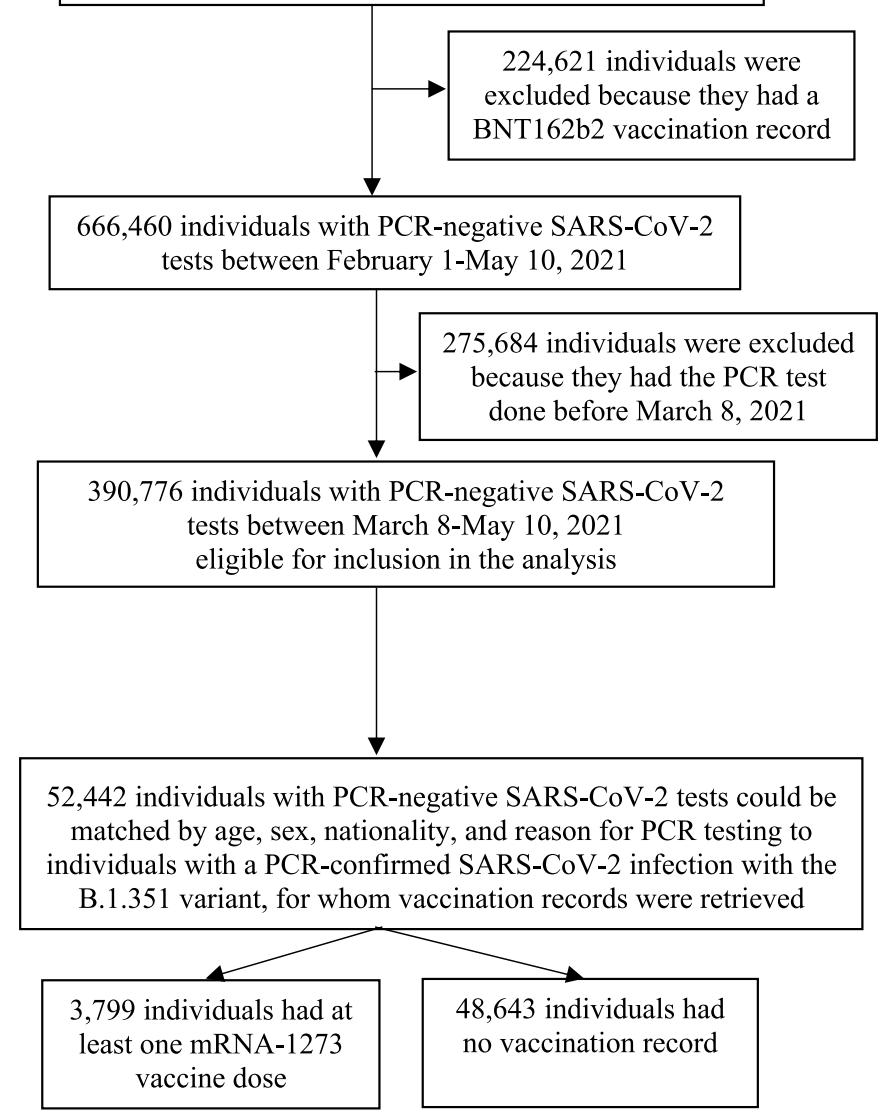

Extended Data Fig. 2 | Flowcharts describing the population selection process to assess vaccine effectiveness against B.1.351. Flowcharts describing the population selection process for investigating mRNA-1273 vaccine effectiveness against infection with the B.1.351 variant. 


\section{C)}

161,788 individuals with a PCR-confirmed SARS-CoV-2 infection between February 1-May 10, 2021

24,547 individuals were excluded because they had a $\mathrm{BNT} 162 \mathrm{~b} 2$ vaccination record

137,241 individuals with a PCR-confirmed SARS-CoV-2 infection between February 1-May 10, 2021

4,906 individuals had a PCR-confirmed SARS-CoV-2 infection that progressed to severe, critical, or fatal disease

4,497 individuals with a PCR-confirmed SARS-CoV-2 infection that progressed to severe, critical, or fatal disease could be matched by age, sex, nationality, and reason for PCR testing to individuals with PCR-negative SARS-CoV-2 tests, for whom vaccination

records were retrieved least one mRNA-1273
182 individuals had at vaccine dose

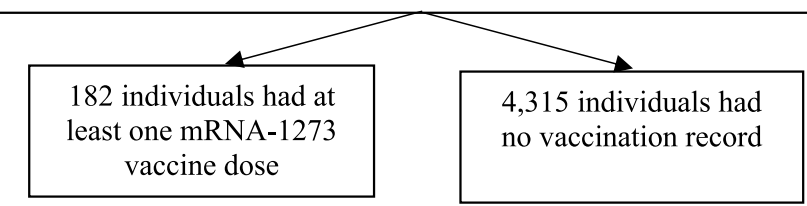

891,081 individuals with PCR-negative SARS-CoV-2 tests between February 1-May 10, 2021

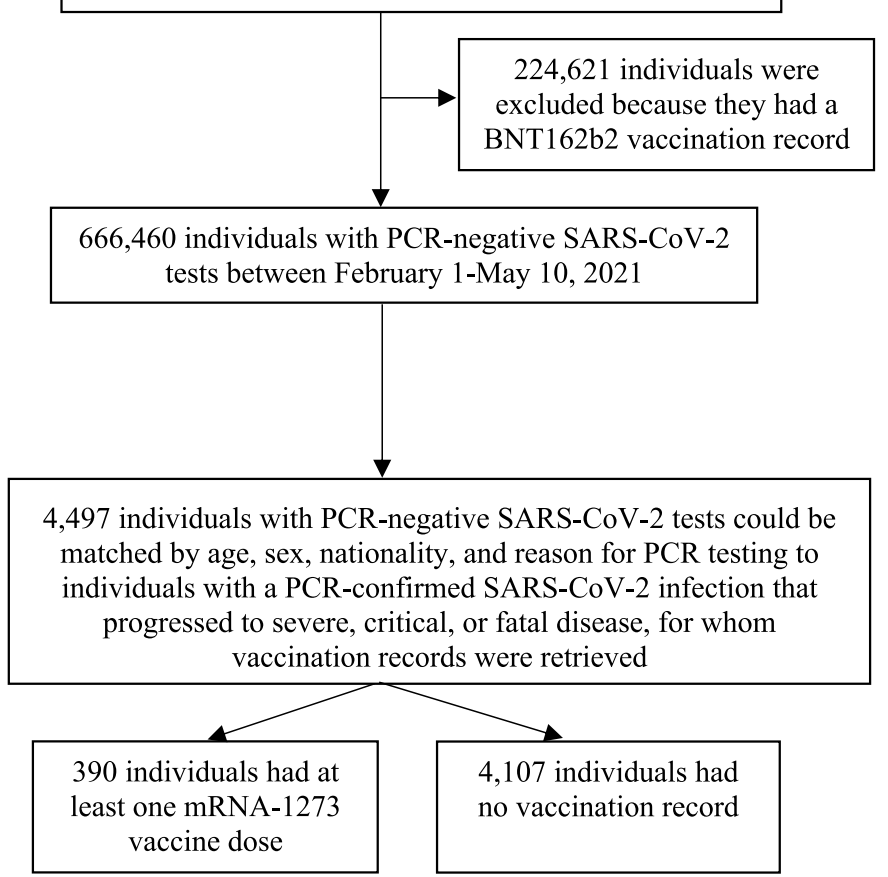

Extended Data Fig. 3 | Flowcharts describing the population selection process to assess vaccine effectiveness against severe, critical, or fatal COVID-19 disease. Flowcharts describing the population selection process for investigating mRNA-1273 vaccine effectiveness against severe, critical, or fatal COVID19 disease. 
A)

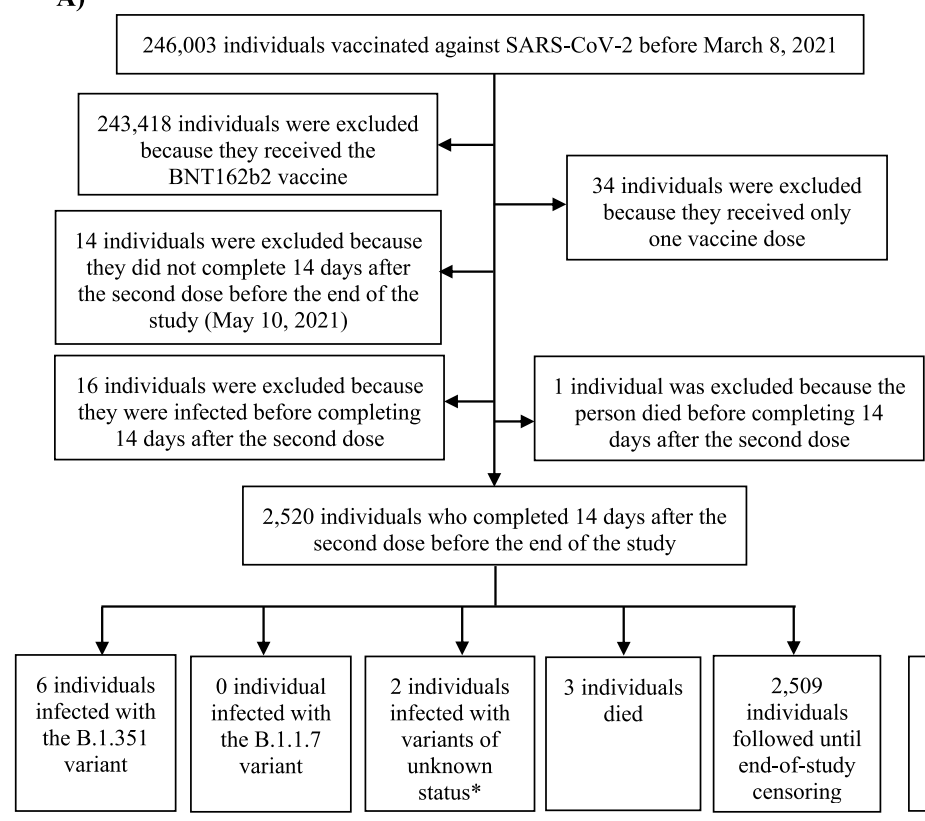

B)

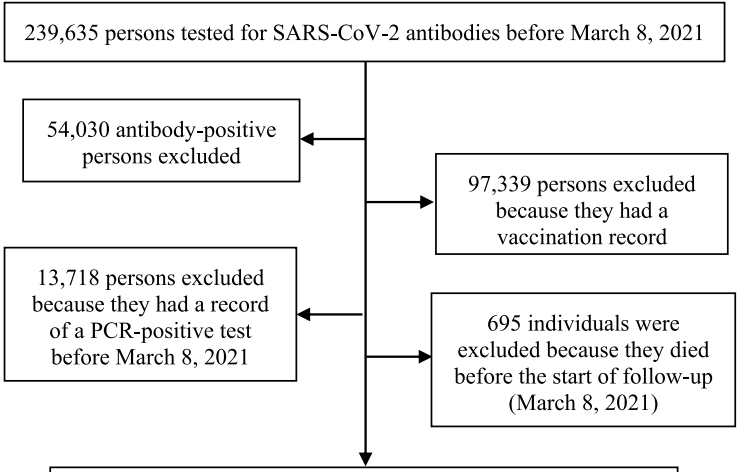

73,853 antibody-negative persons with no record of a prior SARS-CoV-2 infection or vaccination as of March 8, 2021

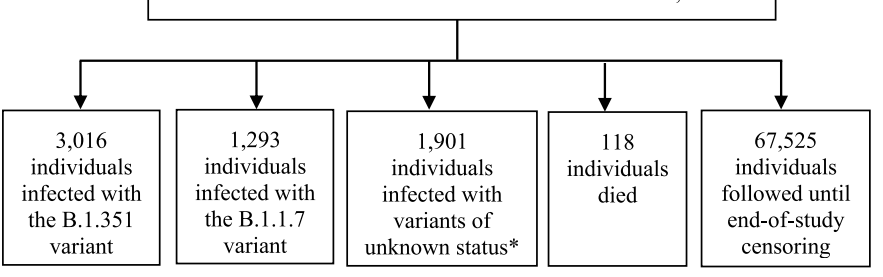

${ }^{*}$ These are likely B.1.351 cases as the B.1.351 variant dominated incidence during this period of follow-up.

Extended Data Fig. 4 | Occurrence of infection in vaccinated and unvaccinated persons. Occurrence of infection in the national cohort of persons vaccinated with the mRNA-1273 vaccine compared to occurrence of infection in the national cohort of those who were antibody-negative. 


\section{Reporting Summary}

Nature Research wishes to improve the reproducibility of the work that we publish. This form provides structure for consistency and transparency in reporting. For further information on Nature Research policies, see our Editorial Policies and the Editorial Policy Checklist.

\section{Statistics}

For all statistical analyses, confirm that the following items are present in the figure legend, table legend, main text, or Methods section.

$\mathrm{n} / \mathrm{a}$ Confirmed

$\bigotimes$ The exact sample size $(n)$ for each experimental group/condition, given as a discrete number and unit of measurement

$\square$ \ A statement on whether measurements were taken from distinct samples or whether the same sample was measured repeatedly

Х The statistical test(s) used AND whether they are one- or two-sided

X Only common tests should be described solely by name; describe more complex techniques in the Methods section.

$\bigotimes$ A description of all covariates tested

Х $\square$ A description of any assumptions or corrections, such as tests of normality and adjustment for multiple comparisons

A full description of the statistical parameters including central tendency (e.g. means) or other basic estimates (e.g. regression coefficient) AND variation (e.g. standard deviation) or associated estimates of uncertainty (e.g. confidence intervals)

$\searrow \square \begin{aligned} & \text { For null hypothesis testing, the test statistic (e.g. } F, t, r \text { ) with confidence intervals, effect sizes, degrees of freedom and } P \text { value noted } \\ & \text { Give } P \text { values as exact values whenever suitable. }\end{aligned}$

Х $\square$ For Bayesian analysis, information on the choice of priors and Markov chain Monte Carlo settings

$\bigotimes \square$ For hierarchical and complex designs, identification of the appropriate level for tests and full reporting of outcomes

$\triangle \square$ Estimates of effect sizes (e.g. Cohen's d, Pearson's $r$ ), indicating how they were calculated

Our web collection on statistics for biologists contains articles on many of the points above.

\section{Software and code}

Policy information about availability of computer code

Data collection Data were available to authors through .csv files

Data analysis Analyses were conducted in STATA/SE 16.1. The commands/code are accessible using URL: https://github.com/IDEGWCMO/Vaccineeffectiveness-code

For manuscripts utilizing custom algorithms or software that are central to the research but not yet described in published literature, software must be made available to editors and

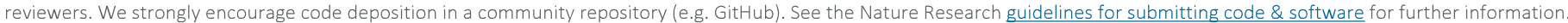

Data

Policy information about availability of data

All manuscripts must include a data availability statement. This statement should provide the following information, where applicable:

- Accession codes, unique identifiers, or web links for publicly available datasets

- A list of figures that have associated raw data

- A description of any restrictions on data availability

The dataset of this study is a property of the Qatar Ministry of Public Health that was provided to the researchers through a restricted-access agreement that prevents sharing the dataset with a third party or publicly. Access to this dataset at any time can be considered through a direct application for data access to her Excellency the Minister of Public Health (https://www.moph.gov.qa/english/Pages/default.aspx). Aggregate data are available within the manuscript and its Supplementary information. 
Please select the one below that is the best fit for your research. If you are not sure, read the appropriate sections before making your selection.

\section{Life sciences study design}

All studies must disclose on these points even when the disclosure is negative.

Sample size

COVID-19 laboratory testing, vaccination, and clinical infection data were extracted from the national, federated SARS-CoV-2 databases that captured all SARS-CoV-2-related data since epidemic onset. The data is based on a national cohort that includes every single individual tested using PCR in Qatar. Sample size varied depending on the definition used for Cases (PCR-positive infected with B.1.1.7 variant, or infected with B.1.351 variant, or developing a severe, critical or fatal infection). Cases (PCR-positive infected with B.1.1.7 variant, or infected with B.1.351 variant, or developing a severe, critical or fatal infection) and controls (PCR-negative) were matched one-to-one by sex, age, nationality, and reason for SARS-CoV-2 polymerase chain reaction (PCR) testing. Only matched pairs were included in the analysis. Given that the sample sizes were based on national cohorts with only individuals that do not fit the eligibility criteria excluded, the sample size for each sub-study can be considered sufficient. Detailed sample sizes can be found in Extended Data 1-4.

Data exclusions Exclusion criteria were specified a priori. For the case control study, these included having a BNT162b2 vaccination record or being tested using PCR outside the study period. For the cohort study, these included having a BNT162b2 vaccination record, being tested using PCR outside the study period, not completing 14 days after the second vaccine dose before the start of the start of the study follow-up, or contracting the infection or dying before the start of the follow-up.

Cases (PCR-positive infected with B.1.1.7 variant, or infected with B.1.351 variant, or developing a severe, critical or fatal infection) and controls (PCR-negative) were matched one-to-one by sex, age, nationality, and reason for SARS-CoV-2 PCR testing. Only matched pairs were included in the analysis and individuals not matched were excluded.

Replication

Sensitivity analyses matching by PCR testing date in addition to age, sex, nationality, and reason for PCR testing; adjusting for calendar week in logistic regression; or additionally adjusting for matching factors, that is sex, age, nationality, and reason for PCR testing, all confirmed results

Randomization Cases and controls were matched one-to-one by sex, age, nationality, and reason for SARS-CoV-2 polymerase chain reaction (PCR) testing. Additionally, to ensure that vaccine effectiveness estimates were not biased by epidemic phase and the gradual roll-out of vaccination during the study, two sensitivity analyses were conducted, first matching by the exact PCR testing date and second by logistic regression to adjust for calendar week16,30. To further ensure control for confounding31,32, a third sensitivity analysis was conducted adjusting additionally for the matching factors in logistic regression, that is sex, age, reason for PCR testing, and nationality.

Blinding

\section{Reporting for specific materials, systems and methods}

We require information from authors about some types of materials, experimental systems and methods used in many studies. Here, indicate whether each material, system or method listed is relevant to your study. If you are not sure if a list item applies to your research, read the appropriate section before selecting a response.

\begin{tabular}{l|l} 
Materials \& experimental syste \\
\hline$n / a$ & Involved in the study \\
$\searrow$ & $\square$ Antibodies \\
$\square$ & $\square$ Eukaryotic cell lines \\
$\searrow$ & $\square$ Palaeontology and archaeology \\
$\square$ & $\square$ Humals and other organisms \\
$\searrow$ & $\square$ Clinical data \\
$\searrow$ & $\square$ Dual use research of concern
\end{tabular}

\begin{tabular}{l|l}
\multicolumn{2}{l}{ Methods } \\
\hline n/a & Involved in the study \\
$\square$ & $\square$ ChIP-seq \\
$\square$ & $\square$ Flow cytometry \\
$\square$ & $\square$ MRI-based neuroimaging
\end{tabular}

\section{Human research participants}

Policy information about studies involving human research participants

Population characteristics

Recruitment
The demographic characteristics of the different study populations can be found in Table 1 and Supplementary Table 2.

This is a retrospective study where COVID-19 laboratory testing, vaccination, clinical infection data, and related demographic details were extracted from the integrated nationwide digital-health information platform that hosts the national, federated SARS-CoV-2 databases. These databases are complete and have captured all SARS-CoV-2-related data since epidemic onset. 
Cases and controls were defined based on analysis for these data. For reasons that remain unclear, among persons 7-13 days after the first dose, risk of infection with B.1.351 was higher compared to those who remained unvaccinated. This might reflect a higher underlying risk of infection, bias due to uncontrolled confounding such as differences in social behavior at or following vaccination, an immunological effect, or an artifact of the estimation method, possibly because the first vaccine dose coincided often with the peak of the B.1.351 wave.

Ethics oversight

The study was approved by the Hamad Medical Corporation and Weill Cornell Medicine-Qatar Institutional Review Boards.

Note that full information on the approval of the study protocol must also be provided in the manuscript. 